\title{
The Kannan Contraction Mapping Theorem for Composition Operators in Metric Spaces and Partially Ordered Metric Spaces
}

\section{Clement Boateng Ampadu}

31 Carrolton Road, Boston, MA 02132-6303, USA

e-mail: drampadu@hotmail.com

\begin{abstract}
In this paper, fixed point theorems of the Kannan type are obtained in the setting of metric space and metric space endowed with partial order, respectively, for self-mappings that are composition operators.
\end{abstract}

\section{Preliminaries}

Definition 1.1 [1]. The composition operator $C_{\psi}$ with symbol $\psi$ is a linear operator defined by the rule

$$
C_{\psi}(f)=f \circ \psi,
$$

where $f \circ \psi$ denotes function composition.

Remark 1.2. Although every real number is a complex number, but not conversely. We assume the domain of the composition operators studied in this paper is $\mathbb{R}$.

Definition 1.3. Let $(\mathbb{R}, d)$ be a metric space, and $C_{\psi}(f)$ be a self map of $(\mathbb{R}, d)$. We say $C_{\psi}(f)$ is a $\psi-f$-Kannan contraction if there exists $k \in\left[0, \frac{1}{2}\right)$ such that the following inequality holds for all $x, y \in \mathbb{R}$,

Received: October 10, 2019; Accepted: December 19, 2019

2010 Mathematics Subject Classification: 47H10, 47B33.

Keywords and phrases: composition operators, Kannan mapping theorem.

Copyright (C) 2020 Clement Boateng Ampadu. This is an open access article distributed under the Creative Commons Attribution License, which permits unrestricted use, distribution, and reproduction in any medium, provided the original work is properly cited. 


$$
d\left(C_{\psi}(f)(x), C_{\psi}(f)(y)\right) \leq k\left[d\left(\psi(x), C_{\psi}(f)(x)\right)\right]+\left[d\left(\psi(y), C_{\psi}(f)(y)\right)\right] .
$$

Remark 1.4. Obviously if $\psi$ is the identity, then $f$ is a Kannan contraction [2], as it satisfies

$$
d(f x, f y) \leq k[d(x, f x)+d(y, f y)]
$$

for some $k \in\left[0, \frac{1}{2}\right)$ and all $x, y \in \mathbb{R}$.

Definition 1.5. Let $C_{\psi}(f)$ be a self-map of $\mathbb{R}$, we say $\psi(b) \in \psi(\mathbb{R})$ is a fixed point of $C_{\psi}(f)$ if $C_{\psi}(f)(b)=\psi(b)$.

Definition 1.6. Let $(\mathbb{R}, d)$ be a metric space. A sequence $\left\{\psi\left(x_{n}\right)\right\}$ in $\psi(\mathbb{R})$ is called convergent, and in particular, converges to $\psi(x) \in \psi(\mathbb{R})$, if $\lim _{n \rightarrow \infty} d\left(\psi\left(x_{n}\right), \psi(x)\right)$ $=0$.

Definition 1.7. Let $(\mathbb{R}, d)$ be a metric space. A sequence $\left\{\psi\left(x_{n}\right)\right\}$ in $\psi(\mathbb{R})$ is called Cauchy, if $\lim _{n, m \rightarrow \infty} d\left(\psi\left(x_{n}\right), \psi\left(x_{m}\right)\right)=0$.

Definition 1.8. We say $(\psi(\mathbb{R}), d)$ is complete, if every Cauchy sequence in $(\psi(\mathbb{R}), d)$ is convergent in $(\psi(\mathbb{R}), d)$.

Using ideas from [3], we introduce the following

Definition 1.9. Let $(X, \preceq)$ be a partially ordered set, and the composition operator $C_{\psi}(f)$ be a self-map of $X$. We say $C_{\psi}(f)$ is non-decreasing if for all $\psi\left(x_{1}\right), \psi\left(x_{2}\right)$ $\in \psi(X)$

$$
\psi\left(x_{1}\right) \preceq \psi\left(x_{2}\right)
$$

$\Rightarrow$

$$
C_{\psi}(f)\left(x_{1}\right) \preceq C_{\psi}(f)\left(x_{2}\right) .
$$

Notation 1.10 [4]. $S$ will denote the class of all functions $\beta: \mathbb{R}^{+} \mapsto[0,1)$ with

(a) $\mathbb{R}^{+}=\{t \in \mathbb{R} \mid t>0\}$,

(b) $\beta\left(t_{n}\right) \rightarrow 0$ implies $t_{n} \rightarrow 0$. 


\section{Kannan Type Fixed Point Theorem}

Theorem 2.1. Let $(\mathbb{R}, d)$ be a metric space. Suppose the composition operator $C_{\psi}(f)$ is a self map of $(\mathbb{R}, d)$ which is a $\psi-f$-Kannan contraction. The fixed point of $C_{\psi}$ is unique, provided $(\psi(\mathbb{R}), d)$ is complete.

Proof. Define the sequence $\left\{\psi\left(y_{n}\right)\right\}$ in $\psi(\mathbb{R})$ by $\psi\left(y_{n+1}\right)=C_{\psi}(f)\left(y_{n}\right)$. Now observe we have the following

$$
\begin{aligned}
d\left(\psi\left(y_{n+1}\right), \psi\left(y_{n+2}\right)\right) & =d\left(C_{\psi}(f)\left(y_{n}\right), C_{\psi}(f)\left(y_{n+1}\right)\right) \\
& \leq k\left[d\left(\psi\left(y_{n}\right), C_{\psi}(f)\left(y_{n}\right)\right)+d\left(\psi\left(y_{n+1}\right), C_{\psi}(f)\left(y_{n+1}\right)\right)\right] \\
& =k\left[d\left(\psi\left(y_{n}\right), \psi\left(y_{n+1}\right)\right)+d\left(\psi\left(y_{n+1}\right), \psi\left(y_{n+2}\right)\right)\right] .
\end{aligned}
$$

From the above, we deduce that

$$
d\left(\psi\left(y_{n+1}\right), \psi\left(y_{n+2}\right)\right) \leq \frac{k}{1-k} d\left(\psi\left(y_{n}\right), \psi\left(y_{n+1}\right)\right) .
$$

Set $h:=\frac{k}{1-k}$, and observe in general for all $n \in \mathbb{N} \cup\{0\}$, we have

$$
d\left(\psi\left(y_{n}\right), \psi\left(y_{n+1}\right)\right) \leq h^{n} d\left(\psi\left(y_{0}\right), \psi\left(y_{1}\right)\right) .
$$

Now consider $n, m \in \mathbb{N} \cup\{0\}$ with $n<m$, and observe we have the following

$$
\begin{aligned}
d\left(\psi\left(y_{n}\right), \psi\left(y_{m}\right)\right) & \leq d\left(\psi\left(y_{n}\right), \psi\left(y_{n+1}\right)\right)+\cdots+d\left(\psi\left(y_{m-1}\right), \psi\left(y_{m}\right)\right) \\
& \leq h^{n} d\left(\psi\left(y_{0}\right), \psi\left(y_{1}\right)\right)+\cdots+h^{m-1} d\left(\psi\left(y_{0}\right), \psi\left(y_{1}\right)\right) \\
& \leq h^{n} d\left(\psi\left(y_{0}\right), \psi\left(y_{1}\right)\right)+h^{n+1} d\left(\psi\left(y_{0}\right), \psi\left(y_{1}\right)\right)+\cdots \\
& =h^{n} d\left(\psi\left(y_{0}\right), \psi\left(y_{1}\right)\right)(1+h+\cdots) \\
& \leq \frac{h^{n}}{1-h} d\left(\psi\left(y_{0}\right), \psi\left(y_{1}\right)\right) .
\end{aligned}
$$

Since $h<1$, if we take limits in the above, we deduce that $\lim _{n, m \rightarrow \infty} d\left(\psi\left(y_{n}\right), \psi\left(y_{m}\right)\right)=0$, and hence $\left\{\psi\left(y_{n}\right)\right\}$ is a Cauchy sequence. By the 
completeness of $(\psi(\mathbb{R}), d)$, there is $\psi\left(a^{*}\right)$ such that $\lim _{n \rightarrow \infty} \psi\left(y_{n}\right)=\psi\left(a^{*}\right)$. Now we show the fixed point exists. Suppose $C_{\psi}(f)\left(a^{*}\right) \neq \psi\left(a^{*}\right)$, and observe we have the following

$$
\begin{aligned}
0 & <d\left(\psi\left(a^{*}\right), C_{\psi}(f)\left(a^{*}\right)\right) \\
& \leq d\left(\psi\left(a^{*}\right), C_{\psi}(f)\left(y_{n}\right)\right)+d\left(\psi\left(C_{\psi}(f)\left(y_{n}\right), C_{\psi}(f)\left(a^{*}\right)\right)\right. \\
& \leq d\left(\psi\left(a^{*}\right), \psi\left(y_{n+1}\right)\right)+k\left[d\left(\psi\left(y_{n}\right), C_{\psi}(f)\left(y_{n}\right)\right)+d\left(\psi\left(a^{*}\right), C_{\psi}(f)\left(a^{*}\right)\right)\right] \\
& =d\left(\psi\left(a^{*}\right), \psi\left(y_{n+1}\right)\right)+k\left[d\left(\psi\left(y_{n}\right), \psi\left(y_{n+1}\right)\right)+d\left(\psi\left(a^{*}\right), C_{\psi}(f)\left(a^{*}\right)\right)\right] .
\end{aligned}
$$

Now taking limits in the above as $n \rightarrow \infty$, we deduce that

$$
d\left(\psi\left(a^{*}\right), C_{\psi}(f)\left(a^{*}\right)\right) \leq k d\left(\psi\left(a^{*}\right), C_{\psi}(f)\left(a^{*}\right)\right) .
$$

Since $1-k \neq 0$ for any $k \in\left[0, \frac{1}{2}\right)$, the above inequality gives

$$
d\left(\psi\left(a^{*}\right), C_{\psi}(f)\left(a^{*}\right)\right)=0 .
$$

It now follows that $\psi\left(a^{*}\right)=C_{\psi}(f)\left(a^{*}\right)$ and so $\psi\left(a^{*}\right)$ is a fixed point of $C_{\psi}(f)$. Finally, we show uniqueness of the fixed point. Suppose $\psi\left(b^{*}\right)$ is any other fixed point of $C_{\psi}(f)$ for which $\psi\left(a^{*}\right) \neq \psi\left(b^{*}\right)$, then we deduce the following

$$
\begin{aligned}
d\left(\psi\left(a^{*}\right), \psi\left(b^{*}\right)\right) & =d\left(C_{\psi}(f)\left(a^{*}\right), C_{\psi}(f)\left(b^{*}\right)\right) \\
& \leq k\left[d\left(\psi\left(a^{*}\right), C_{\psi}(f)\left(a^{*}\right)\right)+d\left(\psi\left(b^{*}\right), C_{\psi}(f)\left(b^{*}\right)\right)\right] \\
& =k\left[d\left(\psi\left(a^{*}\right), \psi\left(a^{*}\right)\right)+d\left(\psi\left(b^{*}\right), \psi\left(b^{*}\right)\right)\right] \\
& =0 .
\end{aligned}
$$

Since the metric is nonnegative, the above inequality implies that $d\left(\psi\left(a^{*}\right), \psi\left(b^{*}\right)\right)=0$, and hence $\psi\left(a^{*}\right)=\psi\left(b^{*}\right)$. It now follows that the fixed point is unique and the proof is complete. 


\section{Application to Partially Ordered Sets}

Theorem 3.1. Let $(\mathbb{R}, \preceq)$ be a partially ordered set, and the composition operator $C_{\psi}(f)$ be a non-decreasing self-map of $\mathbb{R}$ satisfying

$$
\begin{aligned}
d\left(C_{\psi}(f)(x), C_{\psi}(f)(y)\right) \leq \beta & \left(\frac{d\left(\psi(x), C_{\psi}(f)(x)\right)+d\left(\psi(y), C_{\psi}(f)(y)\right)}{2}\right) \\
& \left(\frac{d\left(\psi(x), C_{\psi}(f)(x)\right)+d\left(\psi(y), C_{\psi}(f)(y)\right)}{2}\right)
\end{aligned}
$$

for all $\beta \in S, \psi(x), \psi(y) \in \psi(\mathbb{R})$ such that $\psi(x) \preceq \psi(y)$. Assume $(\psi(\mathbb{R}), d)$ is complete, and suppose further that either

(a) $C_{\psi}(f)$ is continuous

(b) $\psi(\mathbb{R})$ has the property, if a non-decreasing sequence $\left\{\psi\left(x_{n}\right)\right\} \rightarrow \psi(x)$, then

$$
\psi\left(x_{n}\right) \preceq \psi(x)
$$

for all $n \geq 0$.

If there exists $\psi\left(x_{0}\right) \in \psi(\mathbb{R})$ such that $\psi\left(x_{0}\right) \preceq C_{\psi}(f)\left(x_{0}\right)$, then $C_{\psi}(f)$ has a fixed point in $\psi(\mathbb{R})$.

Proof. Let $\psi\left(x_{0}\right)$ be given as in the theorem. Then $\psi\left(x_{0}\right) \preceq C_{\psi}(f)\left(x_{0}\right)$. If $\psi\left(x_{0}\right)=C_{\psi}(f)\left(x_{0}\right)$, then $\psi\left(x_{0}\right)$ is a fixed point. Suppose $\psi\left(x_{0}\right) \prec C_{\psi}(f)\left(x_{0}\right)$. Define $\psi\left(x_{1}\right)=C_{\psi}(f)\left(x_{0}\right), \psi\left(x_{2}\right)=C_{\psi}(f)\left(x_{1}\right)$, and in general,

$$
\psi\left(x_{n}\right)=C_{\psi}(f)\left(x_{n-1}\right)
$$

for all $n \geq 1$. Since $C_{\psi}(f)$ is non-decreasing, we have

$$
\begin{aligned}
\psi\left(x_{0}\right) & \preceq C_{\psi}(f)\left(x_{0}\right) \\
& =\psi\left(x_{1}\right) \\
& \preceq C_{\psi}\left(f^{2}\right)\left(x_{0}\right)
\end{aligned}
$$




$$
\begin{aligned}
& =C_{\psi}(f)\left(x_{1}\right) \\
& =\psi\left(x_{2}\right) \\
& \preceq C_{\psi}\left(f^{3}\right)\left(x_{0}\right) \\
& \preceq \\
& \vdots \\
& \preceq C_{\psi}\left(f^{n}\right)\left(x_{0}\right) \\
& =\psi\left(x_{n}\right) \\
& \preceq C_{\psi}\left(f^{n+1}\right)\left(x_{0}\right) \\
& =\psi\left(x_{n+1}\right) \\
& \preceq
\end{aligned}
$$

If for some $m, \psi\left(x_{m}\right)=\psi\left(x_{m+1}\right)$, then $\psi\left(x_{m}\right)$ is a fixed point of $C_{\psi}(f)$. Hence we assume that $\psi\left(x_{n}\right) \neq \psi\left(x_{n+1}\right)$ for all $n \geq 0$. Since

$$
C_{\psi}\left(f^{n}\right)\left(x_{0}\right)=\psi\left(x_{n}\right)=C_{\psi}\left(f^{n+1}\right)\left(x_{0}\right)=\psi\left(x_{n+1}\right) .
$$

It follows that for all $n \geq 1, \psi\left(x_{n}\right)$ and $\psi\left(x_{n+1}\right)$ are comparable elements. From the inequality of the theorem, we deduce the following

$$
\begin{aligned}
d\left(\psi\left(x_{n+1}\right), \psi\left(x_{n}\right)\right)= & d\left(C_{\psi}(f)\left(x_{n}\right), C_{\psi}(f)\left(x_{n-1}\right)\right) \\
\leq & \beta\left(\frac{d\left(\psi\left(x_{n-1}\right), C_{\psi}(f)\left(x_{n-1}\right)\right)+d\left(\psi\left(x_{n}\right), C_{\psi}(f)\left(x_{n}\right)\right)}{2}\right) \\
& \left(\frac{d\left(\psi\left(x_{n-1}\right), C_{\psi}(f)\left(x_{n-1}\right)\right)+d\left(\psi\left(x_{n}\right), C_{\psi}(f)\left(x_{n}\right)\right)}{2}\right) .
\end{aligned}
$$

By Notation 1.10, we deduce the following

$$
d\left(\psi\left(x_{n+1}\right), \psi\left(x_{n}\right)\right)=d\left(C_{\psi}(f)\left(x_{n}\right), C_{\psi}(f)\left(x_{n-1}\right)\right)
$$




$$
\begin{aligned}
& \leq\left(\frac{d\left(\psi\left(x_{n-1}\right), C_{\psi}(f)\left(x_{n-1}\right)\right)+d\left(\psi\left(x_{n}\right), C_{\psi}(f)\left(x_{n}\right)\right)}{2}\right) \\
& =\left(\frac{d\left(\psi\left(x_{n-1}\right), \psi\left(x_{n}\right)\right)+d\left(\psi\left(x_{n}\right), \psi\left(x_{n+1}\right)\right)}{2}\right) .
\end{aligned}
$$

From the above, we deduce the following

$$
d\left(\psi\left(x_{n+1}\right), \psi\left(x_{n}\right)\right) \leq d\left(\psi\left(x_{n}\right), \psi\left(x_{n-1}\right)\right) .
$$

From the above, we deduce that the sequence $\left\{d\left(\psi\left(x_{n+1}\right), \psi\left(x_{n}\right)\right)\right\}$ is a monotone decreasing sequence of non-negative real numbers, and consequently, there exists $r \geq 0$, such that

$$
\lim _{n \rightarrow \infty} d\left(\psi\left(x_{n+1}\right), \psi\left(x_{n}\right)\right)=r
$$

We claim that $r=0$. If not, observe we have the following

$$
\begin{aligned}
& \frac{d\left(\psi\left(x_{n+1}\right), \psi\left(x_{n}\right)\right)}{d\left(\psi\left(x_{n-1}\right), \psi\left(x_{n}\right)\right)} \\
\leq & \beta\left(\frac{d\left(\psi\left(x_{n-1}\right), C_{\psi}(f)\left(x_{n-1}\right)\right)+d\left(\psi\left(x_{n}\right), C_{\psi}(f)\left(x_{n}\right)\right)}{2}\right) \\
< & 1 .
\end{aligned}
$$

Taking limits in the above as $n \rightarrow \infty$, we deduce the following

$$
\begin{aligned}
1 & =\frac{r}{r} \\
& =\lim _{n \rightarrow \infty} \frac{d\left(\psi\left(x_{n+1}\right), \psi\left(x_{n}\right)\right)}{d\left(\psi\left(x_{n-1}\right), \psi\left(x_{n}\right)\right)} \\
& \leq \lim _{n \rightarrow \infty} \beta\left(\frac{d\left(\psi\left(x_{n-1}\right), C_{\psi}(f)\left(x_{n-1}\right)\right)+d\left(\psi\left(x_{n}\right), C_{\psi}(f)\left(x_{n}\right)\right)}{2}\right) \\
& <1 .
\end{aligned}
$$

It is now clear that

$$
\lim _{n \rightarrow \infty} \beta\left(\frac{d\left(\psi\left(x_{n-1}\right), C_{\psi}(f)\left(x_{n-1}\right)\right)+d\left(\psi\left(x_{n}\right), C_{\psi}(f)\left(x_{n}\right)\right)}{2}\right)=1,
$$


that is,

$$
\lim _{n \rightarrow \infty} \beta\left(\frac{d\left(\psi\left(x_{n-1}\right), \psi\left(x_{n}\right)\right)+d\left(\psi\left(x_{n}\right), \psi\left(x_{n+1}\right)\right)}{2}\right)=1
$$

By Notation 1.10, we deduce the following

$$
\lim _{n \rightarrow \infty} \frac{d\left(\psi\left(x_{n-1}\right), \psi\left(x_{n}\right)\right)+d\left(\psi\left(x_{n}\right), \psi\left(x_{n+1}\right)\right)}{2}=0
$$

which is a contradiction, since we assumed $r>0$. However, the above equation says otherwise, it now follows that the claim is true, that is,

$$
\lim _{n \rightarrow \infty} d\left(\psi\left(x_{n+1}\right), \psi\left(x_{n}\right)\right)=0
$$

Now we show that the sequence $\left\{\psi\left(x_{n}\right)\right\}$ is Cauchy. Suppose not, then given $\varepsilon>0$, we can find sequences $\{m(k)\}$ and $\{n(k)\}$ such that for every natural number $k$, $n(k)>m(k)>k$, and

$$
d\left(\psi\left(x_{m(k)}\right), \psi\left(x_{n(k)}\right)\right) \geq \varepsilon
$$

For each $k>0$, corresponding to $m(k)$, we can choose $n(k)$ to be the smallest integer such that the above inequality holds. It follows for all $k \geq 1$, we have

$$
d\left(\psi\left(x_{m(k)}\right), \psi\left(x_{n(k)-1}\right)\right)<\varepsilon .
$$

Consequently, for $k \geq 1$, we deduce the following

$$
\begin{aligned}
\varepsilon & \leq d\left(\psi\left(x_{m(k)}\right), \psi\left(x_{n(k)}\right)\right) \\
& \leq d\left(\psi\left(x_{m(k)}\right), \psi\left(x_{n(k)-1}\right)\right)+d\left(\psi\left(x_{n(k)-1}\right), \psi\left(x_{n(k)}\right)\right) \\
& \leq \varepsilon+d\left(\psi\left(x_{n(k)-1}\right), \psi\left(x_{n(k)}\right)\right) .
\end{aligned}
$$

Since $\lim _{n \rightarrow \infty} d\left(\psi\left(x_{n+1}\right), \psi\left(x_{n}\right)\right)=0$, if we take limits in the above inequality as $k \rightarrow \infty$, we deduce the following

$$
\lim _{k \rightarrow \infty} d\left(\psi\left(x_{m(k)}\right), \psi\left(x_{n(k)}\right)\right)=\varepsilon
$$


Since

$$
\begin{aligned}
\psi\left(x_{0}\right) & \preceq C_{\psi}(f)\left(x_{0}\right) \\
& =\psi\left(x_{1}\right) \\
& \preceq C_{\psi}\left(f^{2}\right)\left(x_{0}\right) \\
& =C_{\psi}(f)\left(x_{1}\right) \\
& =\psi\left(x_{2}\right) \\
& \preceq C_{\psi}\left(f^{3}\right)\left(x_{0}\right) \\
& \\
& \vdots \\
& \preceq C_{\psi}\left(f^{n}\right)\left(x_{0}\right) \\
& =\psi\left(x_{n}\right) \\
& \preceq C_{\psi}\left(f^{n+1}\right)\left(x_{0}\right) \\
& =\psi\left(x_{n+1}\right) \\
&
\end{aligned}
$$

it follows that $\psi\left(x_{m(k)-1}\right)$ and $\psi\left(x_{n(k)-1}\right)$ are comparable. Since $\psi\left(x_{n}\right)=$ $C_{\psi}(f)\left(x_{n-1}\right)$ and $d\left(\psi\left(x_{m(k)}\right), \psi\left(x_{n(k)}\right)\right) \geq \varepsilon$, we deduce the following from the inequality of the theorem, for all $k \geq 1$,

$$
\begin{aligned}
\varepsilon & \leq d\left(\psi\left(x_{m(k)}\right), \psi\left(x_{n(k)}\right)\right) \\
& =d\left(C_{\psi}(f)\left(x_{n(k)-1}\right), C_{\psi}(f)\left(x_{m(k)-1}\right)\right) \\
& \leq \beta\left(\frac{d\left(\psi\left(x_{n(k)-1}\right), C_{\psi}(f)\left(x_{n(k)-1}\right)\right)+d\left(\psi\left(x_{m(k)-1}\right), C_{\psi}(f)\left(x_{m(k)-1}\right)\right)}{2}\right)
\end{aligned}
$$




$$
\begin{aligned}
& \frac{d\left(\psi\left(x_{n(k)-1}\right), C_{\psi}(f)\left(x_{n(k)-1}\right)\right)+d\left(\psi\left(x_{m(k)-1}\right), C_{\psi}(f)\left(x_{m(k)-1}\right)\right)}{2} \\
< & \frac{d\left(\psi\left(x_{n(k)-1}\right), \psi\left(x_{n(k)}\right)\right)+d\left(\psi\left(x_{m(k)-1}\right), \psi\left(x_{m(k)}\right)\right)}{2} .
\end{aligned}
$$

By the above and Notation 1.10, we deduce the following from the inequality of the theorem

$$
\begin{aligned}
d\left(C_{\psi}(f)(z), \psi\left(x_{n+1}\right)\right)= & d\left(C_{\psi}(f)(z), C_{\psi}(f)\left(x_{n}\right)\right) \\
\leq & \beta\left(\frac{d\left(\psi(z), C_{\psi}(f)(z)\right)+d\left(\psi\left(x_{n}\right), C_{\psi}(f)\left(x_{n}\right)\right)}{2}\right) \\
& \left(\frac{d\left(\psi(z), C_{\psi}(f)(z)\right)+d\left(\psi\left(x_{n}\right), C_{\psi}(f)\left(x_{n}\right)\right)}{2}\right) \\
& \beta\left(\frac{d\left(\psi(z), C_{\psi}(f)(z)\right)+d\left(\psi\left(x_{n}\right), \psi\left(x_{n+1}\right)\right)}{2}\right) \\
& <\frac{d\left(\psi(z), C_{\psi}(f)(z)\right)+d\left(\psi\left(x_{n}\right), \psi\left(x_{n+1}\right)\right)}{2} .
\end{aligned}
$$

Since $\lim _{n \rightarrow \infty} \psi\left(x_{n}\right)=\psi(z)$ and $\lim _{n \rightarrow \infty} d\left(\psi\left(x_{n+1}\right), \psi\left(x_{n}\right)\right)=0$, if we take limits in the above inequality as $n \rightarrow \infty$, we deduce the following

$$
d\left(C_{\psi}(f)(z), \psi(z)\right) \leq \frac{d\left(\psi(z), C_{\psi}(f)(z)\right)}{2} .
$$

The above implies that

$$
C_{\psi}(f)(z)=\psi(z)
$$

It now follows that $C_{\psi}(f)$ has a fixed point in $(\psi(\mathbb{R}), d)$.

Now we show uniqueness of the fixed point via the following 
Theorem 3.2. If in the previous theorem, it is additionally assumed that for every $\psi(y), \psi(z) \in \psi(\mathbb{R})$, there exists $\psi(w) \in \psi(\mathbb{R})$ which is comparable to $\psi(y)$ and $\psi(z)$ and is such that $\psi(w) \preceq C_{\psi}(f)(w)$, then the fixed point in the previous theorem is unique.

Proof. Assume there exists $\psi(z), \psi(y) \in \psi(\mathbb{R})$ which are fixed points of $C_{\psi}(f)$, and consider two cases as follows.

Case 1. $\psi(z)$ and $\psi(y)$ are comparable.

Since $\lim _{n \rightarrow \infty} d\left(\psi\left(x_{n+1}\right), \psi\left(x_{n}\right)\right)=0$, if we take limits in the above inequality as $k \rightarrow \infty$, we deduce the following

$$
\varepsilon \leq \lim _{k \rightarrow \infty}\left(\frac{d\left(\psi\left(x_{n(k)-1}\right), \psi\left(x_{n(k)}\right)\right)+d\left(\psi\left(x_{m(k)-1}\right), \psi\left(x_{m(k)}\right)\right)}{2}\right)=0
$$

which contradicts our assumption that $\varepsilon>0$. It follows that the sequence $\left\{\psi\left(x_{n}\right)\right\}$ is Cauchy. Since $(\psi(\mathbb{R}), d)$ is complete, there is $\psi(z) \in \psi(\mathbb{R})$ such that

$$
\lim _{n \rightarrow \infty} \psi\left(x_{n}\right)=\psi(z)
$$

Now we show the fixed point exists. For this, suppose $(a)$ of the theorem holds, that is, $C_{\psi}(f)$ is continuous, then since $\psi\left(x_{n}\right)=C_{\psi}(f)\left(x_{n-1}\right)$, and $\lim _{n \rightarrow \infty} \psi\left(x_{n}\right)=\psi(z)$, we deduce the following

$$
\begin{aligned}
\psi(z) & =\lim _{n \rightarrow \infty} \psi\left(x_{n}\right) \\
& =\lim _{n \rightarrow \infty} C_{\psi}(f)\left(x_{n-1}\right) \\
& =C_{\psi}(f)\left(\lim _{n \rightarrow \infty} x_{n-1}\right) \\
& =C_{\psi}(f)(z) .
\end{aligned}
$$

Now we assume that $(b)$ of the Theorem holds. Since

$$
\begin{aligned}
\psi\left(x_{0}\right) & \preceq C_{\psi}(f)\left(x_{0}\right) \\
& =\psi\left(x_{1}\right)
\end{aligned}
$$




$$
\begin{aligned}
& \preceq C_{\psi}\left(f^{2}\right)\left(x_{0}\right) \\
& =C_{\psi}(f)\left(x_{1}\right) \\
& =\psi\left(x_{2}\right) \\
& \preceq C_{\psi}\left(f^{3}\right)\left(x_{0}\right) \\
& \preceq \\
& \vdots \\
& \preceq C_{\psi}\left(f^{n}\right)\left(x_{0}\right) \\
& =\psi\left(x_{n}\right) \\
& \preceq C_{\psi}\left(f^{n+1}\right)\left(x_{0}\right) \\
& =\psi\left(x_{n+1}\right) \\
& \preceq
\end{aligned}
$$

and $\lim _{n \rightarrow \infty} \psi\left(x_{n}\right)=\psi(z)$, it follows that $\left\{\psi\left(x_{n}\right)\right\}$ is a nondecreasing sequence in $\psi(\mathbb{R})$ with $\psi\left(x_{n}\right) \rightarrow \psi(z)$, thus $(b)$ of the theorem implies that

$$
\psi\left(x_{n}\right) \preceq \psi(z),
$$

then since

$$
\begin{aligned}
\psi\left(x_{0}\right) & \preceq C_{\psi}(f)\left(x_{0}\right) \\
& =\psi\left(x_{1}\right) \\
& \preceq C_{\psi}\left(f^{2}\right)\left(x_{0}\right) \\
& =C_{\psi}(f)\left(x_{1}\right) \\
& =\psi\left(x_{2}\right) \\
& \preceq C_{\psi}\left(f^{3}\right)\left(x_{0}\right)
\end{aligned}
$$




$$
\begin{aligned}
& \preceq \\
& \vdots \\
& \preceq C_{\psi}\left(f^{n}\right)\left(x_{0}\right) \\
& =\psi\left(x_{n}\right) \\
& \preceq C_{\psi}\left(f^{n+1}\right)\left(x_{0}\right) \\
& =\psi\left(x_{n+1}\right) \\
& \preceq
\end{aligned}
$$

we deduce that $C_{\psi}\left(f^{n}\right)(y)=\psi(y)$ is comparable to $C_{\psi}\left(f^{n}\right)(z)=\psi(z)$, for all $n \geq 1$. It follows from the inequality of the theorem, that we have the following

$$
\begin{aligned}
d(\psi(z), \psi(y)) & =d\left(C_{\psi}\left(f^{n}\right)(z), C_{\psi}\left(f^{n}\right)(y)\right) \\
& \leq \beta\left(\frac{d\left(\psi(z), C_{\psi}\left(f^{n}\right)(z)\right)+d\left(\psi(y), C_{\psi}\left(f^{n}\right)(y)\right)}{2}\right) \\
& \left(\frac{d\left(\psi(z), C_{\psi}\left(f^{n}\right)(z)\right)+d\left(\psi(y), C_{\psi}\left(f^{n}\right)(y)\right)}{2}\right) \\
& =0
\end{aligned}
$$

hence $\psi(z)=\psi(y)$.

Case 2. $\psi(z)$ and $\psi(y)$ are not comparable, then there exists $\psi(w) \in \psi(\mathbb{R})$ which is comparable to $\psi(z)$ and $\psi(y)$. Since $C_{\psi}(f)$ is non-decreasing, it follows that $C_{\psi}\left(f^{n}\right)(w)$ is comparable to $C_{\psi}\left(f^{n}\right)(y)=\psi(y)$ and $C_{\psi}\left(f^{n}\right)(z)=\psi(z)$, for all $n \geq 1$. From the inequality of the theorem, we deduce the following

$$
\begin{aligned}
d(\psi(y), \psi(z)) & \leq d\left(\psi(y), C_{\psi}\left(f^{n}\right)(w)\right)+d\left(C_{\psi}\left(f^{n}\right)(w), \psi(z)\right) \\
& =d\left(C_{\psi}\left(f^{n}\right)(y), C_{\psi}\left(f^{n}\right)(w)\right)+d\left(\left(C_{\psi}\left(f^{n}\right)(w), C_{\psi}\left(f^{n}\right)(z)\right)\right.
\end{aligned}
$$




$$
\begin{aligned}
\leq & \beta\left(\frac{d\left(C_{\psi}\left(f^{n}\right)(w), C_{\psi}\left(f^{n-1}\right)(w)\right)+d\left(C_{\psi}\left(f^{n}\right)(y), C_{\psi}\left(f^{n-1}\right)(y)\right)}{2}\right) \\
& \left(\frac{d\left(C_{\psi}\left(f^{n}\right)(w), C_{\psi}\left(f^{n-1}\right)(w)\right)+d\left(C_{\psi}\left(f^{n-1}\right)(y), C_{\psi}\left(f^{n}\right)(y)\right)}{2}\right) \\
& +\beta\left(\frac{d\left(C_{\psi}\left(f^{n-1}\right)(w), C_{\psi}\left(f^{n}\right)(w)\right)+d\left(C_{\psi}\left(f^{n-1}\right)(z), C_{\psi}\left(f^{n}\right)(z)\right)}{2}\right) \\
& \left(\frac{d\left(C_{\psi}\left(f^{n-1}\right)(w), C_{\psi}\left(f^{n}\right)(w)\right)+d\left(C_{\psi}\left(f^{n-1}\right)(z), C_{\psi}\left(f^{n}\right)(z)\right)}{2}\right) \\
\leq & \frac{d\left(C_{\psi}\left(f^{n}\right)(w), C_{\psi}\left(f^{n-1}\right)(w)\right)+d\left(C_{\psi}\left(f^{n-1}\right)(y), C_{\psi}\left(f^{n}\right)(y)\right)}{2} \\
& +\frac{d\left(C_{\psi}\left(f^{n-1}\right)(w), C_{\psi}\left(f^{n}\right)(w)\right)+d\left(C_{\psi}\left(f^{n-1}\right)(z), C_{\psi}\left(f^{n}\right)(z)\right)}{2} .
\end{aligned}
$$

Since $\psi(w) \preceq C_{\psi}(f)(w)$, then the previous theorem implies $C_{\psi}\left(f^{n}\right)(w) \rightarrow \psi(p)$, where $\psi(p)$ is a fixed point of $C_{\psi}(f)$. Thus, $d\left(C_{\psi}\left(f^{n-1}\right)(w), C_{\psi}\left(f^{n}\right)(w)\right) \rightarrow 0$ as $n \rightarrow \infty$. Thus, taking limits in the above inequality, we get $d(\psi(y), \psi(z)) \leq 0$, that is, $\psi(y)=\psi(z)$ and the proof is finished.

\section{Concluding Remarks and Further Recommendation}

In the present paper we have obtained some Kannan type fixed point theorems for composition operators in metric and partially ordered metric spaces. An interesting direction to consider is the fixed point theory for bivariate composition operators, perhaps inspired by [5] and related works. In this context, we say the bivariate composition operator is a function $C_{\psi}(F): \mathbb{R} \times \mathbb{R} \mapsto \mathbb{R}$ defined by the rule

$$
C_{\psi}(F)(x, y):=F(\psi(x), \psi(y))
$$




\section{References}

[1] Wikipedia: The Free Encyclopedia, Composition operator. https://en.wikipedia.org/wiki/Composition_operator

[2] R. Kannan, Some results on fixed points, Bull. Calcutta Math. Soc. 60 (1968), 71-76. https://doi.org/10.2307/2316437

[3] A. Amini-Harandi and H. Emami, A fixed point theorem for contraction type maps in partially ordered metric spaces and application to ordinary differential equations, Nonlinear Anal. TMA 72(5) (2010), 2238-2242. https://doi.org/10.1016/j.na.2009.10.023

[4] M. A. Geraghty, On contractive mappings, Proc. Amer. Math. Soc. 40 (1973), 604-608. https://doi.org/10.2307/2039421

[5] D. Guo and V. Lakshmikantham, Coupled fixed points of nonlinear operators with applications, Nonlinear Anal. 11 (1987), 623-632.

https://doi.org/10.1016/0362-546X(87)90077-0 